Proceedings of the 2011 Winter Simulation Conference

S. Jain, R.R. Creasey, J. Himmelspach, K.P. White, and M. Fu, eds.

\title{
DESIGN OF A MANUFACTURING FACILITY LAYOUT WITH A CLOSED LOOP CONVEYOR WITH SHORTCUTS USING QUEUEING THEORY AND GENETIC ALGORITHMS
}

\author{
Vernet Lasrado \\ Dima Nazzal \\ University of Central Florida \\ Department of Industrial Engineering and Management Systems \\ 4000 Central Florida Blvd. \\ Orlando, FL 32816-2993 USA
}

\begin{abstract}
Most current manufacturing facility layout problem solution methods aim at minimizing the total distance traveled, the material handling cost, and/or the time spent in the system (based on distance traveled at a specific speed). The methodology proposed in this paper solves the looped layout design problem for a looped layout manufacturing facility with a looped conveyor material handling system with shortcuts by using the operational performance metric, i.e. the work-in-process on the conveyor in a manufacturing facility, as the design criterion.
\end{abstract}

\section{INTRODUCTION}

A typical manufacturing facility (MF) consists of a production system (PS) and a material handling system (MHS). The PS consists of numerous operational cells henceforth referred to as a cell or cells. The literature generally refers to a cell in the PS as a machine, a facility, a station, a collection of stations, a department, a bay, etc. The MHS transfers the manufactured units, henceforth referred to as loads or jobs, from one cell to another. The types of MF layouts used in MHS design include single row, multi row, closed loop layout (Kusiak and Heragu 1987), and open field layout (Loiola et al. 2007). We will focus on the layout of a MF, i.e. the manufacturing facility layout problem (MFLP) for a closed loop layout. We will refer to these as the looped layout MF (LLMF) and the looped layout design problem (LLDP), following the nomenclature introduced in Nearchou (2006).

We begin by defining MFLP as an optimization problem whose solution determines the mostefficient physical organization of the cells in a PS considering an objective, such as minimizing the material handling cost (MHC), distance traveled, or time spent in the system (Benjaafar, Heragu and Irani 2002). Previous MFLP formulations have tended to ignore the impact of a facility's layout on the operational performance of the MF, i.e. the work-in-process (WIP), the throughput, or the cycle time. Benjaafar (2002) shows that traditional MF design criteria can be a poor indicator of an MF's operational performance. Bozer and Hsieh (2005) also support this argument. Kouvelis, Kurawarwala and Gutierrez (1992) state that "the use of 'optimality' with respect to a design objective, such as the minimization of the material handling cost, is discriminating." Benjaafar (2002) argues that the MF's operational performance is contingent upon the congestion in the MF, which is a function of its capacity and variability. Hence, it is imperative that the objective of the MFLP captures the impact of the facility layout on an MF's operational performance. This can be achieved, for example, by setting the objective of the MFLP to minimize the WIP in the MF (Benjaafar 2002; Fu \& Kaku 1997; Kouvelis \& Kiran 1991; Raman, Nagalingam and Gurd 2008). However, despite the presence of conveyor systems in high volume manufacturing facilities, 


\section{Lasrado and Nazzal}

to the best of our knowledge no methods have been proposed that generate the layout by minimizing the WIP in a LLMF with a closed loop conveyor (CLC) as the MHS.

This research proposes a methodology that addresses the development of a facility layout for an LLMF with a looped conveyor material handling system (LCMHS) that can have shortcuts across it, i.e. an LLDP, with the objective of minimizing the WIP.

\section{DESCRIPTION OF THE FACILITY}

A brief description of the LLMF given below entails the assumptions, definitions and characteristics of the LLMF. Figure 1 presents the LLMF.

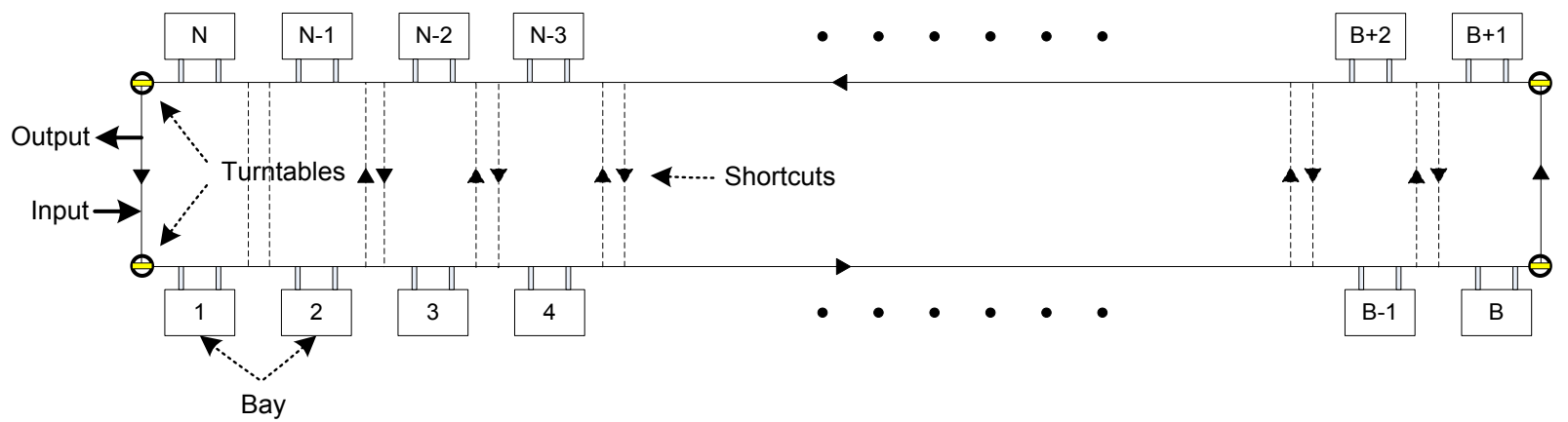

Figure 1: Layout of the Facility

We assume that the number of machines required and their groupings are predetermined. The machines may be used individually or as a group, but in either case, we refer to them as cells. We assign $M$ cells $(\mathrm{i}=1,2,3, \ldots, M)$ to $N$ locations $(\mathrm{j}=1,2,3, \ldots, N)$ where $M \leq N$. If $M<N$ dummy cells $(M+1, M+2, \ldots, N)$ are introduced as recommended by Hillier and Connors (1966). There is one entry point (loading cell) and one exit point (shipping cell) to the LLMF. At the entry point $(i=0)$ products are delivered/loaded into the plant and at the exit point $(i=N+1)$ products are shipped/unloaded from the plant.

The routing for each product through the LLMF is known and is deterministic. Products may visit each cell more than once. We use the decomposition method presented in Whitt (1983) to determine the internal flows between the cells, with the internal flows represented by $\lambda_{i j}$ where the product travels from cell $i(i=1,2, \ldots, N)$ to cell $j(j=1,2, \ldots, N)$ using an LCMHS and $\lambda_{i i}=0$.

The LLMF shown in Figure 1 is a job shop with interconnected bays/cells that each consist of a group of machines or individual machines, an automated material handling system (AMHS) which is an LCMHS with no load recirculation (i.e. infinite buffer at unloading station from the conveyor), an input station (where new jobs are introduced to the LLMF) and an output station (where completed jobs are moved from the LLMF), and shortcuts as shown in Figure 2.

The MF has a variable demand, for loads. The loads can backtrack to cells (revisit facilities) and bypass cells in the MF. Each cell has a loading/unloading station where loads are loaded to/unloaded from the conveyor.

We characterize the arrival process to cell $i$ by an independently distributed random variable with an average interarrival time $\left(1 / \lambda_{i}\right)$ and a squared coefficient of variation $\left(c_{a i}^{2}\right)$, and the service process at each cell by an independently distributed random variable with a mean service time $\left(\tau_{i}\right)$ and a squared coefficient of variation $\left(c_{s i}^{2}\right)$.

In the LLMF, a shortcut can be placed after each cell $\mathrm{i}$ in the direction of flow such that it is before the next cell $i+1$ and before the corresponding shortcut from the opposite side of the conveyor. Figure 2 illustrates the two sides of the conveyor. Cell $\mathrm{p}$ and cell $\mathrm{q}$ are on one side and cell $\mathrm{r}$ and cell $\mathrm{s}$ are on the 


\section{Lasrado and Nazzal}

opposite side. The shortcut $\mathrm{p}$ (arc 'eh') after cell $\mathrm{p}$ is placed in the direction of flow before cell $\mathrm{q}$ and before the corresponding shortcut $\mathrm{r}$ ( $\mathrm{arc}$ ' $\mathrm{gf}$ ') from cell $\mathrm{r}$ on the opposite side of the conveyor.

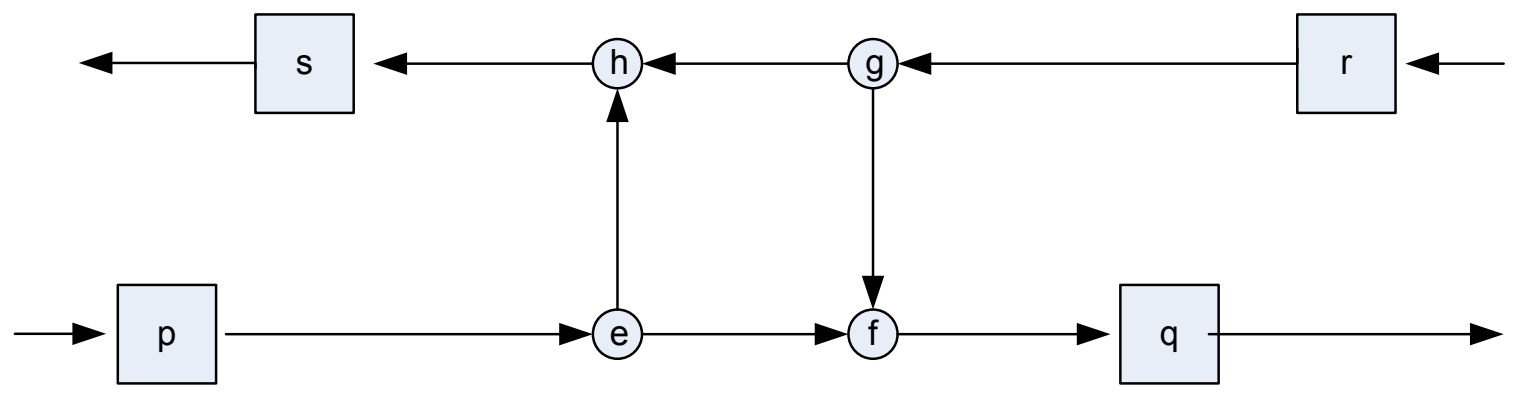

Figure 2: Shortcut diagram

When a cell is the last cell on its side of the conveyor in the direction of flow, we always place a shortcut after that cell (the short wall of the conveyor that connects the two sides shown in Figure 1.)

\section{ANALYTICAL MODEL OF THE WIP}

\subsection{The Traveling WIP on the Conveyor}

The following analysis provides an estimate for the expected traveling WIP on the conveyor without considering any turntables as described in Bozer and Hsieh (2005). The conveyor travels at a constant speed; move requests follow a Poisson process; loading and unloading stations have unlimited capacity; and the conveyor is continuous with no turning delays (no turntables).

Consider that the conveyor is a series of nodes with a set of segments $(S)$ connecting two nodes to form a network of nodes where the segments are the length of the conveyor connecting two cells. A window $(Y)$ is the length of a conveyor defined to hold at most one job. Therefore, for a segment $i$ with length $d_{i}$, the number of windows $\left(w_{i}\right)$ is given by

$$
w_{i}=\frac{d_{i}}{\Upsilon}
$$

Let $\alpha_{i}$ be the average number of loads per time unit on segment $i$ and $s$ be the distance based speed of the conveyor. The window based speed of the conveyor $(v)$ with respect to the window size in terms of windows per time unit is given by

$$
v=\frac{s}{\Upsilon}
$$

This representation of the inherent speed of the conveyor is general as it account for non-unity window sizes. As long as the conveyor system is stable as described in Bozer and Hsieh (2004), the probability that segment $i$ is occupied $\left(q_{i}\right)$ is given by

$$
q_{i}=\frac{\alpha_{i}}{v}
$$

The expected traveling WIP $\left(W I P_{\text {CONV }}\right)$ on the conveyor is given by

$$
W I P_{C O N V}=\sum_{i \in S} w_{i} q_{i}
$$




\section{Lasrado and Nazzal}

\subsection{Proposed Methodology for Estimating the WIP at Input (loading) Stations}

We model the loading station as a $\mathrm{G} / \mathrm{G} / \mathrm{m}$ queue where if $\mathrm{m}>1$, the loading station has multiple loading robots. In general, the service time is the amount of time the load waits at the front of the queue, i.e. the head of the line (HOL), before being loaded onto the conveyor. Each load at the loading station is served for either a Type 1 or a Type 2 service distribution.

We assume that the time the robot takes to transfer the load to the conveyor (drop-off time) is negligible in comparison to the conveyor cycle time, which we define as the time to move one window. It can be argued that in many practical situations the load transfer time onto the conveyor is not negligible with respect to the conveyor cycle time. In this case, we propose to adjust the window size ( $\Upsilon$ ) so as to reduce the distance based speed $(s)$ such that the conveyor cycle time is greater than the load drop-off time. In this case, the proposed method can be applied to estimate the WIP on the conveyor and the WIP at the input stations. Another approach would be to modify the service time distributions in Sections 3.2.1 and 3.2.2 to account for the time the robot takes to transfer the load; this is an area of future research.

\subsubsection{Type 1 Service Distribution}

When a load arrives at an empty input station queue:

Event 1 The load waits for the residual conveyor cycle time The Uniform Distribution with the range $[0,1 / v]$ is used to model event 1

Event 2 The load waits for the first unoccupied window

The Geometric distribution with probability $\phi_{i}$ ( $\phi_{i}$ is further discussed in $\S 3.2 .4$ ) is used to model the expected number of windows until the arrival of the first empty window. Multiplied by the conveyor cycle time, it is used to model event 2

We assume that both events are independent of one another. For each cell $\mathrm{i}$, the expected service time for service type $1, E\left(S T 1_{i}\right)$, is given by

$$
E\left[S T 1_{i}\right]=\frac{1}{2 v}+\frac{\phi_{i}}{1-\phi_{i}} \cdot \frac{1}{v}
$$

For each cell $\mathrm{i}$, the variance for service type $1, \operatorname{Var}\left[S T 1_{i}\right]$, is given by

$$
\operatorname{Var}\left[S T 1_{i}\right]=\frac{1}{12 v^{2}}+\frac{\phi_{i}}{\left(1-\phi_{i}\right)^{2}} \cdot \frac{1}{v^{2}}
$$

\subsubsection{Type 2 Service Distribution}

When the load arrives at a non-empty input station queue:

Event 2 The load waits for the first unoccupied window

The Geometric distribution with probability $\phi_{i}$ ( $\phi_{i}$ is further discussed in $\S 0$ ) is used to model the expected number of windows until the first empty window. Multiplied by the conveyor cycle time, it is used to model event 2

For each cell $i$, the expected service time for service type $2, E\left(S T 2_{i}\right)$, is given by 


$$
E\left[S T 2_{i}\right]=\frac{\phi_{i}}{1-\phi_{i}} \cdot \frac{1}{v}
$$

For each cell $i$, the variance for service type $2, \operatorname{Var}\left[S T 2_{i}\right]$, is given by

$$
\operatorname{Var}\left[S T 2_{i}\right]=\frac{\phi_{i}}{\left(1-\phi_{i}\right)^{2}} \cdot \frac{1}{v^{2}}
$$

\subsubsection{Expected WIP at input stations}

For each cell $i$, the WIP at the input stations as per Welch (1964) is given by

$$
\begin{aligned}
E\left(W I P_{I N P}\right)_{i}= & \frac{E\left[S T 2_{i}\right]}{1-\lambda_{i}\left[E\left[S T 2_{i}\right]-E\left[S T 1_{i}\right]\right]} \\
+ & \frac{\lambda_{i}\left[\operatorname{Var}\left[S T 1_{i}\right]+E\left[S T 1_{i}\right]^{2}-\left(\operatorname{Var}\left[S T 2_{i}\right]+E\left[S T 2_{i}\right]^{2}\right)\right]}{2\left\{1-\lambda_{i}\left[E\left[S T 2_{i}\right]-E\left[S T 1_{i}\right]\right]\right\}} \\
& +\frac{\lambda_{i}\left[\operatorname{Var}\left[S T 2_{i}\right]+E\left[S T 2_{i}\right]^{2}\right]}{2\left\{1-\lambda_{i} E\left[S T 2_{i}\right]\right\}}
\end{aligned}
$$

Substituting equations (4)-(7) in equation (9), we get

$$
E\left(W I P_{I N P}\right)_{i}=\frac{2 \lambda_{i} \phi_{i}}{\left(2 v+\lambda_{i}\right)\left(1-\phi_{i}\right)}+\frac{\lambda_{i}^{2}\left(1+\phi_{i}-2 \phi_{i}^{2}\right)}{3 v\left(2 v+\lambda_{i}\right)\left(1-\phi_{i}\right)^{2}}+\frac{\lambda_{i}^{2}\left(\phi_{i}+\phi_{i}^{2}\right)}{2 v\left[v-\phi_{i}\left(v+\lambda_{i}\right)\right]\left(1-\phi_{i}\right)}
$$

\subsubsection{A note on the adjusted probability}

The adjusted probability $\phi_{i}$ is the probability that a load arriving at the input station in segment $i$ sees an occupied window. $\phi_{i}$ is developed using numerical experiments in a manner similar to the G/G/1 approximation by Kraemer and Langenbach-Belz (1976) and reported by Shanthikumar and Buzacott (1980).

We observe that using the probability that the segment is occupied, i.e. $q_{i}$, as the probability of success for the Geometric distribution underestimates the number of Bernoulli trials estimated until the first success. We also observe that under high utilization the adjustment required to $q_{i}$ is minimal, whereas under low utilization the adjustment to $q_{i}$ is significant. Intuitively, under high utilization, since the conveyor is almost completely occupied $q_{i}$ approaches $\phi_{i}$, and theoretically at a utilization of $1, q_{i}=\phi_{i}$. With this in mind, for each cell i a simple metric for $\phi_{i}$ is given by

$$
\phi_{i}=q_{i}\left(2-\rho_{i}\right)
$$

where the utilization for each cell $\mathrm{i}$ is given by

$$
\rho_{i}=\frac{\lambda_{i}+\alpha_{i}}{v}
$$




\section{Lasrado and Nazzal}

Thus, (11) satisfies all of the observances described above: under low utilizations the adjustment factor is significant; under high utilizations the adjustment factor is minimal; and when $\rho_{i}$ is $1, \phi_{i}=q_{i}$.

\section{OPTIMIZATION MODEL}

The total cost function is given by

$$
Z=c\left[E\left(W I P_{I N P}\right)+E\left(W I P_{C O N V}\right)\right]+C \cdot \# \text { Shortcuts }
$$

Let $\Omega$ be the ratio of the cost of installing the shortcut to the cost of a single unit of production. The effective cost per production unit is given by

$$
x=E\left(W I P_{I N P}\right)+E\left(W I P_{C O N V}\right)+\Omega \cdot \# \text { Shortcuts }
$$

This method is employed by Hong et al. (2011) to bypass the problem of determining the price of a unit of production and the price of installing a shortcut. We focus on the formulation by permutations since it is the most commonly used formulation and extends itself well to formulations with very complicated objective functions. According to Hillier and Connors (1966) and Loiola et al. (2007), if $S_{N}$ is the set of all permutations of $N$ variables, given that each permutation $(\pi)$ represents a unique layout of the MF, i.e. a unique assignment of $M$ cells to $N$ locations, then $\pi \in S_{N}$. For an LLMF, the LLDP with a LCMHS is presented below using the formulation by permutations: the objective function as shown in (15) represents the total WIP in the system for a permutation $\pi$. (16) is included to ensure only feasible permutations if the LLMF is considered to resolve the LLDP.

$$
\begin{array}{ll}
\operatorname{Min}_{\pi \in S_{N}} & E\left(W I P_{I N P}\right)+E\left(W I P_{C O N V}\right)+\Omega \cdot \# \text { Shortcuts }\left.\right|_{\pi} \\
\text { s.t. } & \rho_{\pi}<1
\end{array}
$$

where

$$
\rho_{\pi}=\left.\max \left(\rho_{i}\right)\right|_{\pi}
$$

\subsection{Solution Algorithm}

We employ a genetic algorithm (GA) to solve the LLDP as described in (15) and (16). In essence, for each permutation $\pi$, we use the greedy heuristic described by Johnson et al. (2009) to determine the number of shortcuts, and then compute the fitness (objective) value from (14). In this manner, the fitness value from (15) for each permutation $\pi \in S_{N}$ reflects the benefit (if any) of including the shortcuts.

\section{APPLICATION}

SEMATECH (2002) developed a virtual fabrication model of a general semiconductor manufacturing facility. Using its flow rates between cells as the test data for our proposed model, we multiply them by 4 (to stress test the system), and set the conveyor speed to $0.305 \mathrm{ft} / \mathrm{sec}$. The SEMATECH (2002) had a determined layout, as shown by the unboxed numbers in Figure 3. The original SEMATECH simulation model layout's fitness value is 945.72 . We execute the GA to obtain the best layout with no shortcuts 
(Figure 3). The fitness (objective) value is 526.06, a $44.37 \%$ reduction over the original SEMATECH layout.

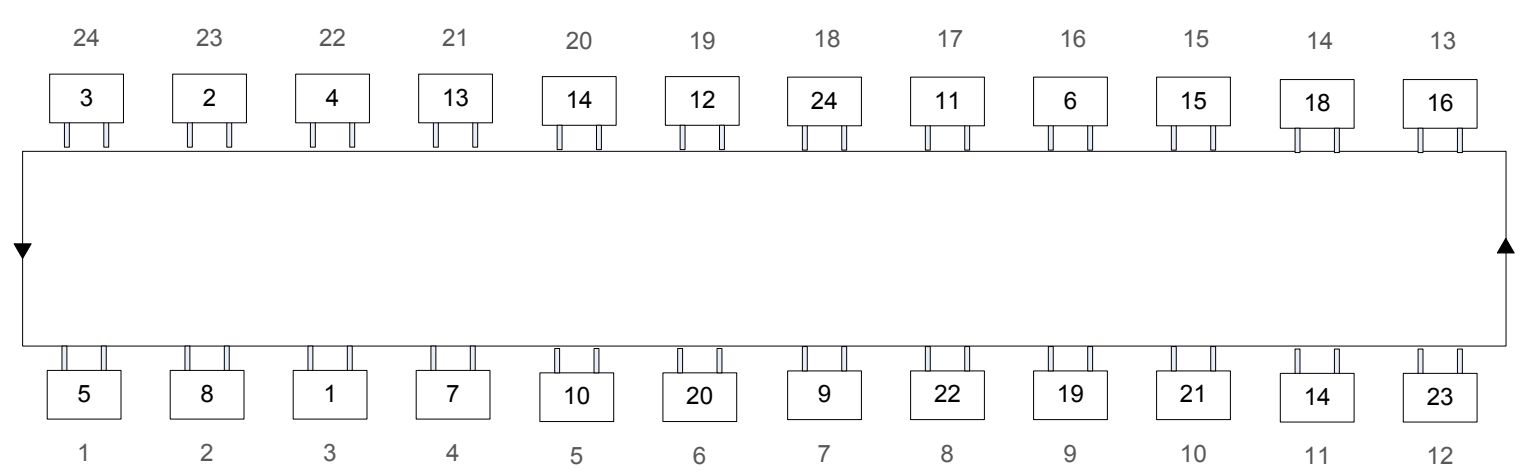

Figure 3: Layout for MF with no shortcuts

Here, the numbers in the boxes represent the cells (machines/facilities) and the lighter unboxed numbers represent the locations. For example, cell 5 is assigned to location 1, cell 8 to location 2, and so on. Henceforth for simplicity, the pictographic layout representations omit the location information.

Figure 4 shows the best layout with $\Omega=10$ with a fitness (objective) value of 329.21 .

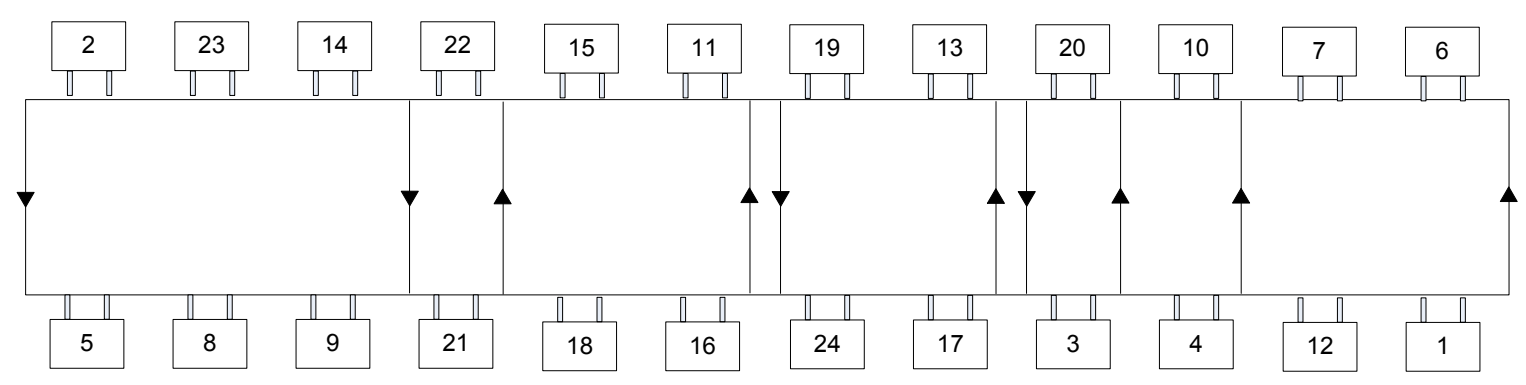

Figure 4: Layout for MF with shortcuts and $\Omega=10$

Figure 5 shows the best layout with $\Omega=25$ with a fitness (objective) value of 404.39 .

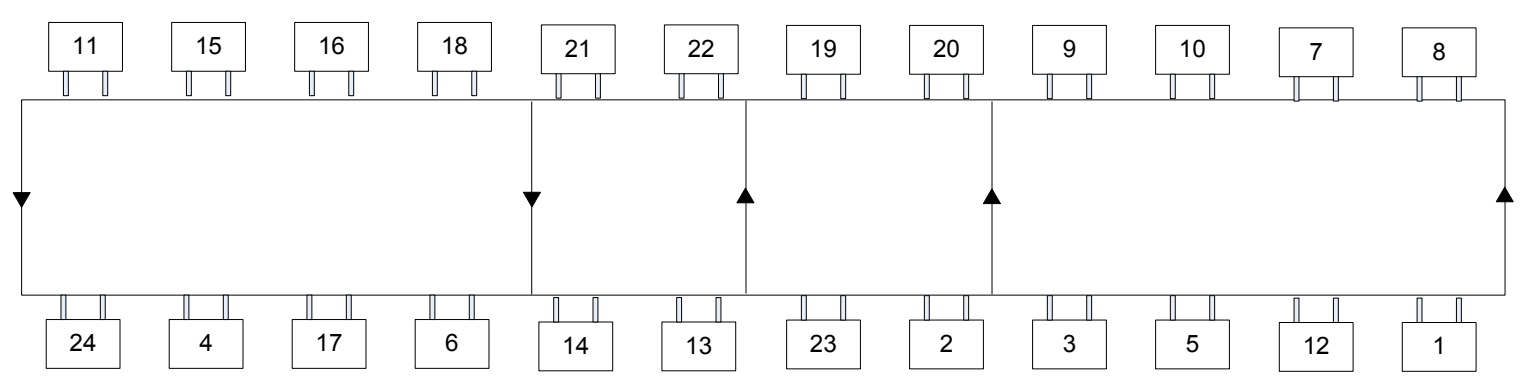

Figure 5: Layout for MF with shortcuts and $\Omega=25$

Comparing cell layouts gives interesting results. For example, when no shortcuts are allowed, a specific layout of the MF is prescribed, i.e. a specific assignment of cells to locations is prescribed. We want to find the fitness value when $\Omega=10$ or $\Omega=25$. Using this layout we determine the set of shortcuts using the Greedy algorithm (Johnson et al., 2009) and the fitness value when $\Omega=10$ or $\Omega=25$. Next, we com- 


\section{Lasrado and Nazzal}

pare both the layout of cells from each solution used in the other scenarios and the solutions themselves. This comparative exercise illustrates that merely determining the best set of shortcuts given a current layout will not guarantee the lowest WIP.

Table 1: Test Problem $-\Omega=10$ versus Other Layouts

\begin{tabular}{c|cc}
\hline Solution & Distance & Fitness \\
\hline Original SEMATECH & 135871.15 & 501.91 \\
$\Omega=10$ & $\mathbf{9 2 5 0 4 . 7 8}$ & $\mathbf{3 2 9 . 2 1}$ \\
$\Omega=25$ & 96103.70 & 357.58 \\
No Shortcut & 102973.87 & 391.31 \\
\hline
\end{tabular}

As expected, the ' $\Omega=10$ ' solution has the lowest fitness values and the lowest total distance travelled by the units of production. Examining the solutions in Table 1 we observe the impact of the combined solution algorithm (layout and shortcuts simultaneously) versus the two step process (first best layout and then best shortcuts). For example, by using the no shortcuts solution we can obtain a semi-optimal solution. In many ways, this is analogous to a global solution versus a local solution for a non-linear optimization problem.

The results of the comparisons are presented below (see Appendix B for the pictographic comparison).

Table 2: Test Problem $-\Omega=25$ versus Other Layouts

\begin{tabular}{c|cc}
\hline Solution & Distance & Fitness \\
\hline Original SEMATECH & 146476.36 & 622.36 \\
$\Omega=10$ & $\mathbf{1 0 3 9 7 7 . 1 1}$ & 415.64 \\
$\Omega=25$ & 108616.48 & $\mathbf{4 0 4 . 3 9}$ \\
No Shortcut & 122270.28 & 440.69 \\
\hline
\end{tabular}

As expected, the ' $\Omega=25$ ' solution has the lowest fitness values. Interestingly, the best fitness solution does not have the lowest total distance travelled by the units of production. If the decision criterion is to minimize the distance travelled, we would select the $\Omega=10$ solution. However, this solution does not have the lowest cost with respect to WIP and conveyor costs. Again, it is evident that the lowest distance travelled solution does not guarantee the lowest cost with respect to the WIP and conveyor cost.

\section{CONCLUSIONS}

Traditionally, a solution algorithm is used to determine the best layout, followed by another solution algorithm (for example, Johnson et al. (2009) or Hong et al. (2011)) to determine the best set of shortcuts. However, the benefits of the shortcuts are only seen for the best non shortcut based layout. The methodology proposed in this paper gives the benefits of the shortcuts for every layout encountered and thereby guaranteeing an equivalent or better solution compared to the traditional method. In other words, it is possible to see the impact and benefit of the combined solution algorithm (layout and shortcuts simultaneously) versus the two step process (first best layout and then best shortcuts). 


\section{Lasrado and Nazzal}

\section{APPENDIX A: LAYOUT WITH $\Omega=10$ VERSUS OTHERS (FIGURE 6)}

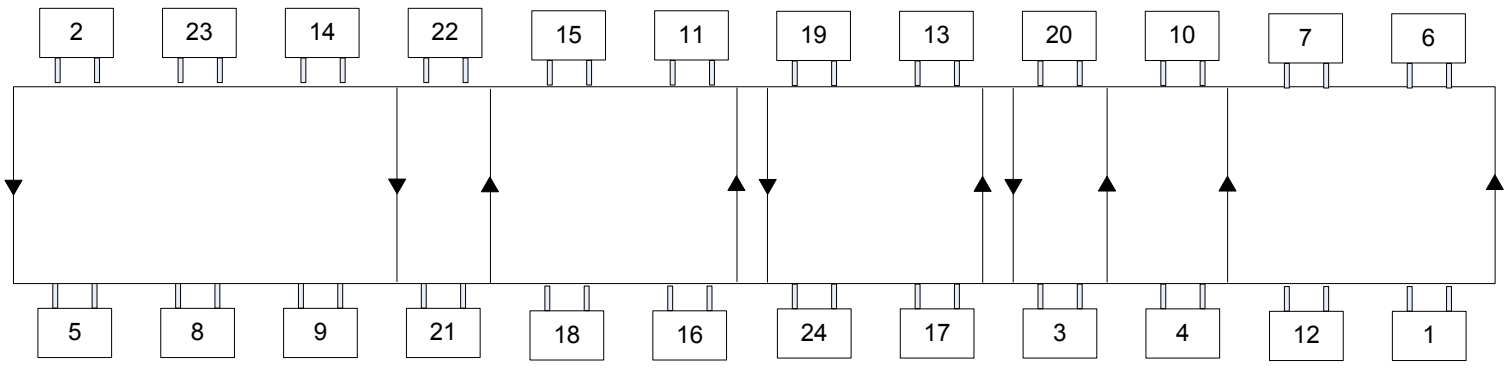

(a) Best Current Solution for $\Omega=10$ (Fitness $=329.21$ )

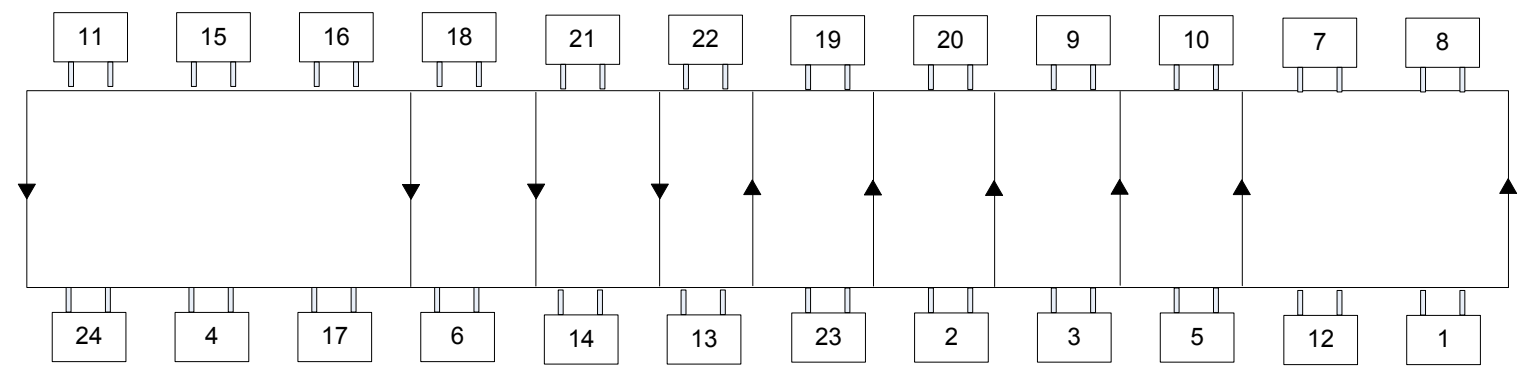

(b) Best Solution from $\Omega=25$ applied to $\Omega=10$ (Fitness $=357.58$ )

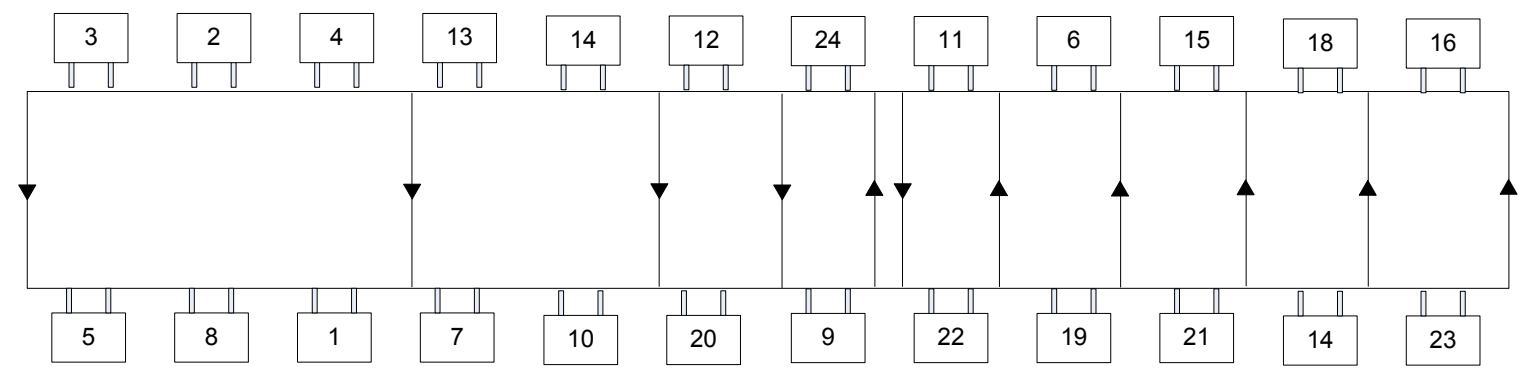

(c) Best Solution from no shortcut applied to $\Omega=10($ Fitness $=391.31)$

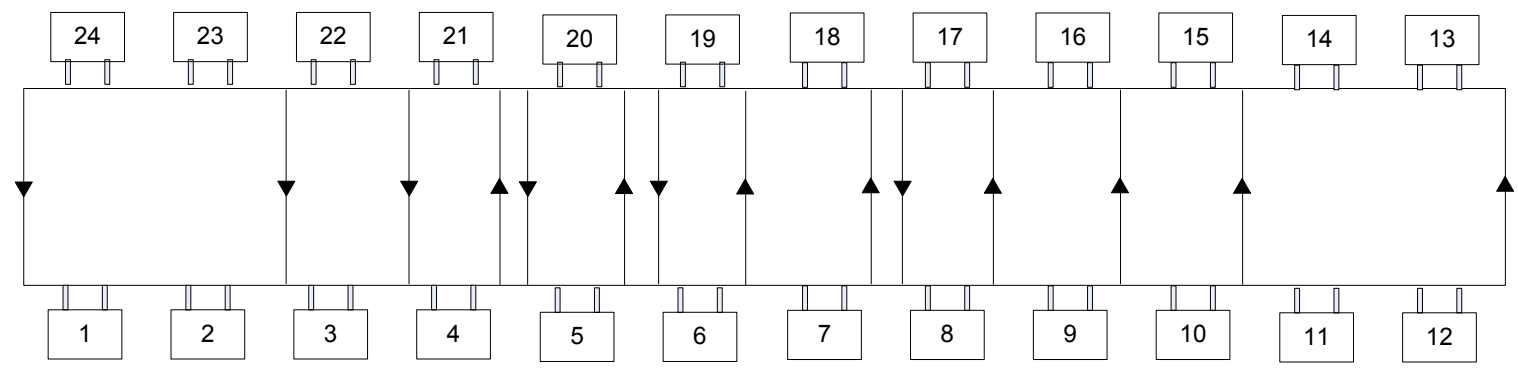

(d) Original SEMATECH Layout applied to $\Omega=10($ Fitness $=501.91)$ 
APPENDIX B: LAYOUT WITH $\Omega=25$ VERSUS OTHERS (FIGURE 7)

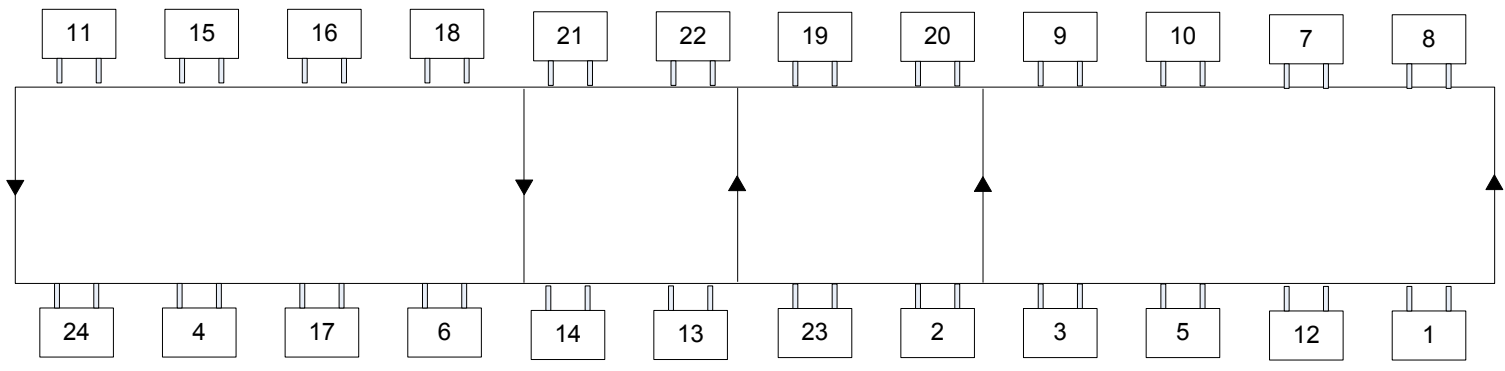

(a) Best Current Solution for $\Omega=25$ (Fitness $=404.39$ )

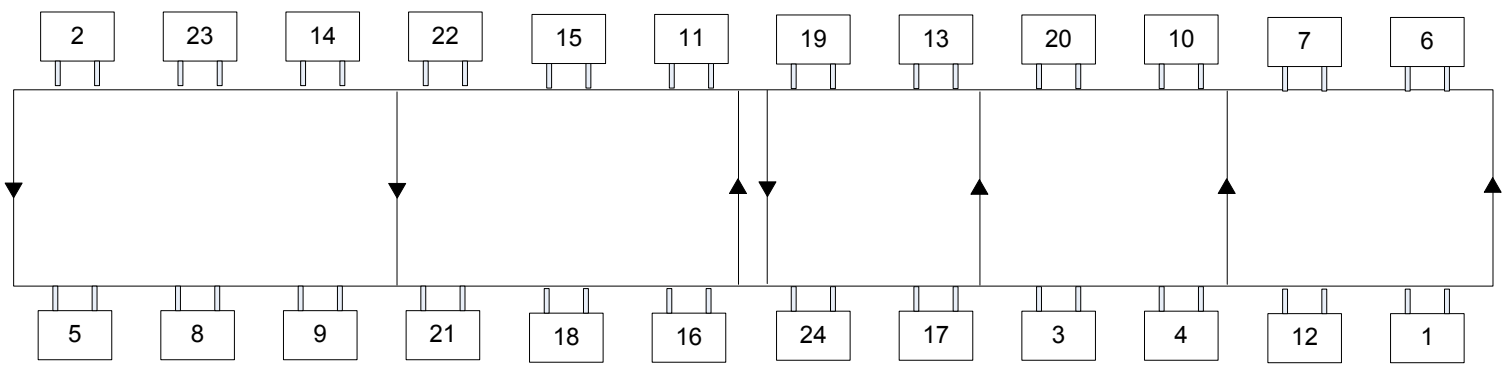

(b) Best Solution from $\Omega=10$ applied to $\Omega=25$ (Fitness $=415.64$ )

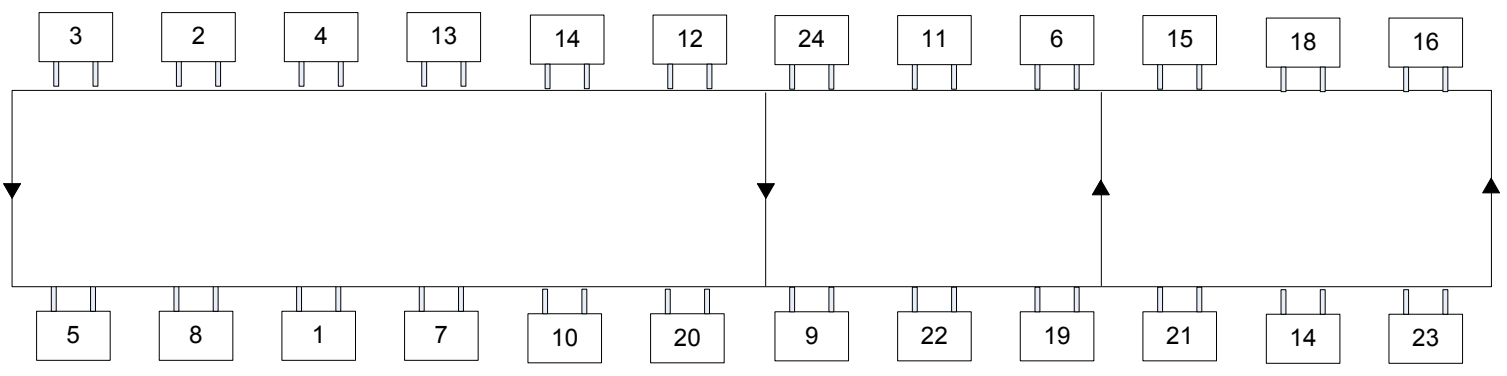

(c) Best Solution from no shortcut applied to $\Omega=25$ (Fitness $=440.69$ )

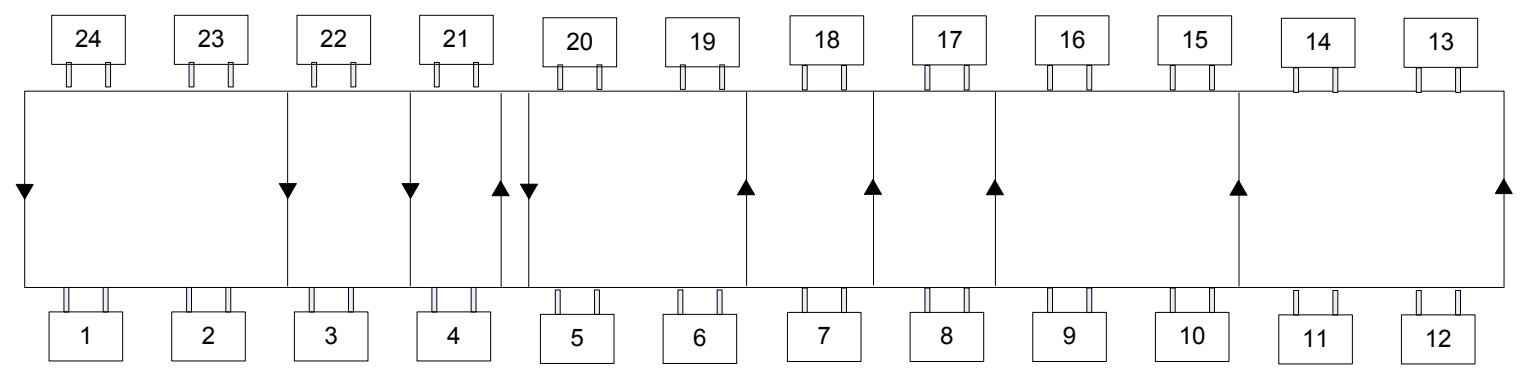

(d) Original SEMATECH Layout applied to $\Omega=25$ (Fitness $=622.36$ ) 


\section{Lasrado and Nazzal}

\section{REFERENCES}

Benjaafar, S. 2002. "Modeling and analysis of congestion in the design of facility layouts." Management Science, 48 (5): 679-704.

Benjaafar, S., S. Heragu and S. Irani. 2002. "Next generation factory layouts: research challenges and recent progress." Interfaces, 32 (6): 58-76.

Bozer, Y. and Y. Hsieh. 2004. "Expected waiting times at loading stations in discrete-space closed-loop conveyors." European Journal of Operational Research, 155 (2): 516-532.

- 2005. "Throughput performance analysis and machine layout for discrete-space closed-loop conveyors." IIE Transactions, 37 (1): 77-89.

Fu, M., and B. Kaku. 1997. "Minimizing work-in-process and material handling in the facilities layout problem." IIE Transactions, 29 (1): 29-36.

Hillier, F. and M. Connors. 1966. "Quadratic assignment problem algorithms and the location of indivisible facilities." Management Science, 13: 42-57.

Hong, S., A. Johnson, H. Carlo, D. Nazzal and J. Jimenez. 2011. "Optimising the location of crossovers in conveyor-based automated material handling systems in semiconductor wafer fabs." International Journal of Production Research, 1-28.

Johnson, A., H. Carlo, J. Jimenez, D. Nazzal and V. Lasrado. 2009. A greedy heuristic for locating crossovers in conveyor-based AMHS in wafer fabs. In Proceedings of the 2009 Winter Simulation Conference, edited by M. D. Rossetti, R. R. Hill, B. Johansson, A. Dunkin and R. G. Ingalls, 1667-1676. Piscataway, New Jersey: Institute of Electrical and Electronics Engineers, Inc.

Kouvelis, P., A. Kurawarwala and G. Gutierrez. 1992. "Algorithms for robust single and multiple period layout planning for manufacturing systems." European Journal of Operational Research, 63 (2): 287-303.

Kraemer, W., and M. Langenbach-Belz. 1976. "Approximate Formulae for the Delay in the Queueing System GI/G/1." In Congress Book, 235-1/8. Melbourne, Australia.

Kusiak, A., and S. Heragu. 1987. "The facility layout problem." European Journal of Operational Research, 29 (3): 229-251.

Loiola, E., N. de Abreu, P. Boaventura-Netto, P. Hahn, and T. Querido. 2007. "A survey for the quadratic assignment problem." European Journal of Operational Research, 176 (2): 657-690.

Nearchou, A. 2006. "Meta-heuristics from nature for the loop layout design problem." International Journal of Production Economics 101 (2): 312-328.

Raman, D., S. Nagalingam, and B. Gurd. 2008. "A genetic algorithm and queuing theory based methodology for facilities layout problem." International Journal of Production Research, 47 (20): 1-25.

SEMATECH. 2002. Fab simulation modeling software. http://ismi. sematech.org/modeling/simulation/index.htm\#models.

Shanthikumar, J., and J. Buzacott. 1980. "On the approximations to the single server queue." International Journal of Production Research, 18 (6): 761.

Welch, P. 1964. "On a generalized M/G/1 queuing process in which the first customer of each busy period receives exceptional service." Operations Research, 12 (5): 736-752.

Whitt, W. 1983. “The queueing network analyzer.” Bell System Technical Journal, 62 (9): 2779-2815.

\section{AUTHOR BIOGRAPHIES}

VERNET LASRADO is a PhD Candidate in the Department of Industrial Engineering and Management Sciences at the University of Central Florida and is the recipient of the Provost Fellowship. He obtained his BSc. degree from The H. Milton Stewart School of Industrial and Systems Engineering at Georgia Tech, Atlanta, Georgia, in 2006, and his MSc. Degree from the Department of Industrial Engineering and Management Sciences at the University of Central Florida. His research interests include the layout, mod- 
eling and analysis of manufacturing systems and the application of evolutionary algorithms to solve optimization problems. His email address is vernet.lasrado@ucf.edu.

DIMA NAZZAL is an Assistant Professor in the Department of Industrial Engineering and Management Systems at the University of Central Florida, Orlando, Florida. She obtained her Ph.D. degree from The H. Milton Stewart School of Industrial and Systems Engineering at Georgia Tech, Atlanta, Georgia, in 2006, and her M.S. in Industrial Engineering from The University of Central Florida in 2001. She earned her B.S. in Industrial Engineering from The University of Jordan in 1998. She is a member of INFORMS and IIE. Her research interests are in analytical modeling of manufacturing and logistics systems, and incorporating environmental sustainability into design of production and distribution systems. Her email address is dima.nazzal@ucf.edu. 